\title{
SmartLB: Proposta de um balanceador de carga para redução de tempo de execução de aplicações em ambientes paralelos
}

\author{
Vinicius R. S. dos Santos ${ }^{1}$, Edson L. Padoin ${ }^{1,2}$, \\ Philippe O. A. Navaux ${ }^{2}$, Jean-François Méhaut ${ }^{3}$ \\ ${ }^{1}$ Universidade Reg. do Noroeste do Estado do Rio G. do Sul (UNIJUI) - Ijuí - RS - Brasil \\ \{vinicius.ribas, padoin\}@unijui.edu.br \\ ${ }^{2}$ Universidade Federal do Rio Grande do Sul (UFRGS) - Porto Alegre - RS - Brasil \\ navauxdinf.ufrgs.br \\ ${ }^{3}$ Universite Grenoble Alpes - Grenoble - França \\ jean-francois.mehauteimag.fr
}

\begin{abstract}
This article presents a new load balancer to reduce the execution time and energy consumption of parallel applications. The balancer algorithm collects system information and uses it to take balancing splits. For implementation, the parallel programming model $\mathrm{CHARM++}$ was used. The preliminary results showed a reduction of the execution time of up to $40.9 \%$ and up to $47.7 \%$ in energy consumption.
\end{abstract}

Resumo. Este artigo apresenta um novo balanceador de carga para redução do tempo de execução e consumo de energia de aplicações paralelas. $O$ algoritmo do balanceador coleta informações do sistema e as utiliza para tomar decisões de balanceamento. Para implementação foi utilizado o modelo de programação paralela $\mathrm{CHARM++.} \mathrm{Os} \mathrm{resultados} \mathrm{preliminares} \mathrm{apresentaram} \mathrm{redução} \mathrm{do} \mathrm{tempo} \mathrm{de} \mathrm{execução}$ de até $40,9 \%$ e até $47,7 \%$ no consumo de energia.

\section{Introdução}

Cada vez mais aplicações científicas são simuladas em ambientes paralelos. Tais simulações computacionais representam atualmente um percentual significativo no total das demandas por processamento nos sistemas de HPC e, somente são possíveis com o emprego de programação paralela. Com paralelismo o processamento das aplicações é dividido em partes e executado em paralelo nas unidades de processamento disponíveis nos atuais de sistemas de HPC.

Para atender às grandes demandas de processamento, diferentes arquiteturas paralelas tem sido projetas e construídas empregando processadores compostos de múltiplas unidades de processamento. No entanto, a maioria das aplicações paralelas apresentam características, como comportamento dinâmico, que acabam gerando desbalanceamento de cargas, ou excessiva comunicação entre os objetos. Tais características dificultam a eficiente utilização dos sistemas de computação que geralmente possuem unidades de processamento homogêneos.

Nesse sentido, este artigo apresenta a proposta de uma nova estratégia de balanceamento de carga denominado SMARTLB. Nossa proposta almeja a redução do tempo total de execução e do consumo de energia por meio da migração de tarefas considerando a carga computacional média das unidades de processamento também denominados de cores ou núcleos. 
O trabalho está organizado da seguinte forma. A Seção 2 discute o estado da arte das tecnologias a serem utilizadas e os trabalhos relacionados. A Seção 3 apresenta a estratégia, a metodologias de implementação e o algoritmo do balanceador de carga proposto. A Seção 4 descreve a metodologia e o ambiente utilizado na implementação e execução dos testes. Resultados alcançados são discutidos na Seção 5, seguidos das Conclusões e Trabalhos Futuros.

\section{Trabalhos Relacionados}

Muitas pesquisas tem sugerido o emprego de balanceamento de carga para reduzir o tempo de execução e o consumo de energia das plataformas quando executam aplicações paralelas.

O balanceamento de carga é uma técnica utilizada para a distribuição uniforme da carga computacional e da comunicação entre todos os cores ou processadores de uma máquina paralela. Estas estratégias podem ser empregadas quando as tarefas são criadas ou periodicamente durante a execução das aplicações paralelas [Zheng et al. 2010].

Estratégias como GREEDYLB, REFINELB e RANDCENTLB são consideradas centralizadas, pois fazem decisões de balanceamento de carga em um único processador. Elas coletam informações de carga de toda a máquina em um core específico, o qual que executa um processo de decisão sequencial com base nessas informações [Zheng et al. 2010]. Outras estratégias, visando uma melhor escalabilidade quando aplicadas em sistemas de grande escala, adotam abordagens distribuídas onde os processadores distribuídos tomam decisões usando sua visão local do sistema. Nessas estratégias, os processadores trocam dados de balanceamento de carga apenas entre os vizinhos, como forma de descentralizar o processo de decisão [Cybenko 1989].

\section{Balanceador de Carga SmartLB}

Nossa proposta, busca reduzir o overhead de balanceamento a partir da implementação de melhorias nas estratégias utilizadas nos algoritmos GREEDYLB, REFINELB e AVERAGELB. Utilizando uma abordagem centralizada almeja-se alcançar balanceamento de carga levando em consideração a média aritmética das cargas de cada unidade de processamento, reduzindo o número total de migrações, o tempo de execução e consequentemente o consumo de energia.

\subsection{Metodologias de Implementação}

Para implementar a estratégia de balanceamento de carga proposta foi utilizado o ambientes de programação paralela $\mathrm{CHARM++}$. A escolha por este ambiente foi motivada pela sua madura estrutura de balanceamento de carga, a qual permite tanto a criação de novos balanceadores de carga, quanto a utilização dos balanceadores de carga disponibilizados pelo ambiente para comparações de resultados.

Dentre as informações armazenadas pelo SMARTLB destacam-se o número total de tarefas, a carga de cada tarefa, o número total de núcleos e a carga total de cada núcleo. Estes dados são utilizados pela estratégia na tomada de decisões para definir quais tarefas devem ser migradas e qual o novo mapeamento de cada tarefa para que seja mitigado o desequilíbrio de carga.

\subsection{Algoritmo}

O SMARTLB adota um threshold para definir um limite de desbalanceamento de carga aceitável. Caso o desbalanceamento seja maior que o threshold previamente definido, o algoritmo busca atingir o balanceamento levando em consideração a diferença de carga entre os 
cores com carga acima da média e abaixo da média. Quando o balanceador é chamado, ele usa informações disponibilizadas pelo $\mathrm{CHARM}++$ e calcula as cargas computacionais de cada core.

Quando a estratégia é aplicada, o algoritmo (linhas 1 a 3 ) busca informações sobre a quantidade de objetos mapeados em cada core e suas cargas para calcular o core com maior carga $(P M)$, o core com menor carga $(P m)$ e a média das cargas (avg).

Tendo computado estes valores, a estratégia (linha 4) compara o desbalanceamento entre a carga do core mais carregado e o core menos carregado com o threshold . Caso essa razão for menor, o desbalanceamento de carga presente é menor do que o limite aceitável, assim nenhuma tarefa é migrada. Por outro lado, se a razão for maior que o threshold definido, o balanceador busca tarefas (linha 6 ) dos cores com cargas acima da média (linha 8) e realiza a migração desta tarefa para o core menos carregado (linha 13).

A estratégia (linha 11) também verifica antes de migrar se esta migração não deixará o core que recebe a tarefa com carga acima da média, para isso testa se a carga da tarefa é menor ou igual a diferença entre o core subcarregado e core atual.

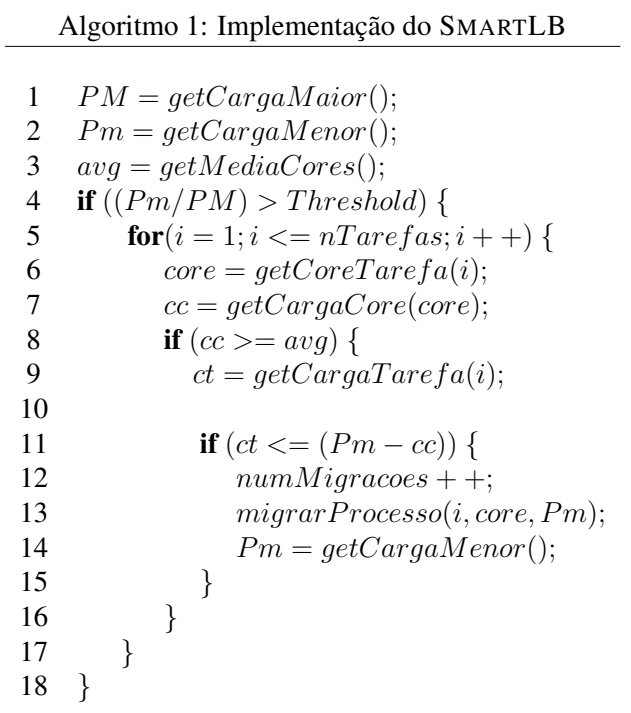

\section{Metodologia}

Nossos experimentos foram realizados em uma plataforma Altix UV 2000 projetada pela SGI. A plataforma é composta por 24 nós NUMA. Cada nó tem um processador Intel Xeon E5-4640 modelo Sandy Bridge-EP x86-64 com 8 núcleos físicos de 2,40 GHz.

Cada núcleo do Intel Xeon E5-4640 tem memórias caches L1 de 32 KB para instrução e $32 \mathrm{~KB}$ para dados e $256 \mathrm{~KB}$ de cache L2. Todos os 8 núcleos compartilham uma memória cache de 20 MB L3. Cada nó possui 32 GB de memória DDR3, que é compartilhada com outros nós de uma forma cc-NUMA através do NUMAlink6 da SGI. Em geral, esta plataforma tem 192 núcleos físicos e 768 GB de memória DDR3, dos quais foram utilizados somente 24, um core de cada processador.

A plataforma executa um sistema operacional SUSE Linux Enterprise com o kernel 3.0.101 - 0.29. Todos os benchamrks e balanceadores de carga foram compilados com GCC 4.8.2. A versão do CHARM++ utilizada para implementação foi a 6.5.1. 
Para analisar o desempenho, a demanda de potência, o consumo de energia foi utilizada a ferramenta EMonDaemon [Padoin et al. 2014]. Esta ferramenta possibilita a coleta e análise do tempo de execução e da demanda de potência de cada processador durante a execução dos testes. Para realização dos testes a ferramenta EMonDaemon foi configurada para realizar medições de demanda de potência do processador a cada $1 \mathrm{~s}$.

Cada um dos testes realizados neste trabalho foram repetidos 10 vezes, para atingirmos um erro relativo menor que $5 \%$ e $95 \%$ de confiança estatística para uma Student's t-distribution. Entre cada um dos testes foi deixado o sistema em idle por no mínimo 20 segundos, de modo que a demanda de potência do sistema se estabilizasse.

\subsection{Benchmarks}

Para avaliar o desempenho, demanda de potência e o consumo de energia do balanceador de carga proposto foram selecionados três benchmarks. Eles foram escolhidos devido à sua variada gama de padrões de comunicação e características da carga de trabalho. A descrição de cada benchmarks é apresentada na sequência :

- lb test: benchmark que apresenta desequilíbrio de carga onde pode-se usar diferentes padrões de comunicação. Neste artigo, foi utilizado um grafo de comunicação aleatória.

- kNeighbor: benchmark iterativo onde cada tarefa se comunica com "k"outras tarefas em cada etapa ou iteração. Seu padrão de comunicação é um anel.

- ComprehensiveBench: benchmark sintético configurável usado para criar aplicativos iterativos com diferentes parâmetros, como o número de tarefas, iterações, gráfico de comunicação, carga de tarefas e tamanhos de mensagens.

A Tabela 1 apresenta as características dos benchmarks e parâmetros utilizados em nossos experimentos. Diferentes frequências de balanceamento de carga foram escolhidas para diferentes aplicações, a fim de estabelecer um equilíbrio entre os benefícios das tarefas de remapeamento e os custos de mover tarefas entre cores bem como a computação de um novo mapeamento de tarefas. Nos testes com o balanceador de carga proposto foi adotado um threshold de valor igual a $5 \%$.

Tabela 1. Parâmetros de entrada dos Benchmarks.

\begin{tabular}{lccc}
\hline Benchmarks & Tarefas & Iterações & Frequência de LB \\
\hline lb_test & 200 & 150 & 10 \\
kNeighbor & 1600 & 50 & 10 \\
ComprehensiveBench & 85 & 50 & 5 \\
\hline
\end{tabular}

\subsection{Balanceadores de carga}

Os resultados de tempo de execução e a economia de energia alcançados com o BC proposto foram comparados com os balanceadores de carga GREEDYLB, REFINELB e AVERAGELB.

\section{Resultados}

Nesta seção são apresentados as avaliações de desempenho, as redução do tempo de execução, do consumo de energia alcançadas com o emprego do nosso balanceador de carga SMARTLB na plataforma experimental apresentada na última seção. 
Na Figura 1 são apresentados os tempos de execução dos testes realizados com o benchmark $l b \_t e s t$ e kNeighbor para diferentes quantidades de tarefas.

Figura 1. Tempos de execução mensurados durante a execução

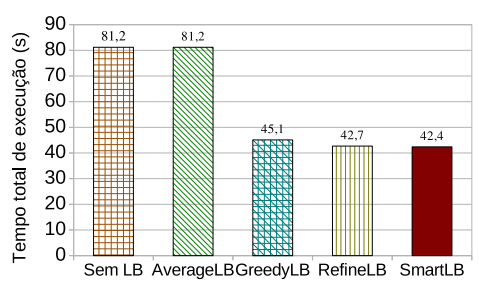

(a) $l b \_t e s t$

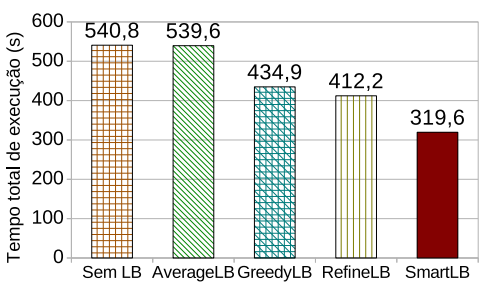

(b) kNeighbor

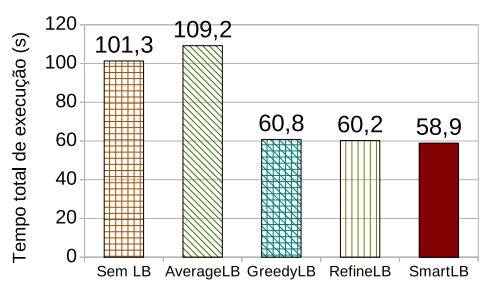

(c) ComprehensiveBench

O BC SMARTLB apresentou melhor desempenho para ambos os benchmarks testados. Para lb_test com 200 tarefas o SMARTLB conseguiu reduzir o tempo de 81,2 para 42, 4 segundos, o que representa uma redução de 47,8\% em relação à execução sem balanceador.

Quando foi executado o benchmark kNeighbor, o SMARTLB conseguiu uma redução significativa no tempo de execução. Com 1600 tarefas o tempo total de execução foi reduzido de 540,8 para 319,6 segundos, o que representa uma redução de $40,9 \%$ em relação à execução sem balanceador. Para este benchmark, o SMARTLB apresentou tempos de $22,4 \%$ menor que o balanceador REFINELB e de 26, $5 \%$ menor que o balanceador GREEDYLB.

Figura 2. Consumo de energia mensurado durante a execução dos benchmarks

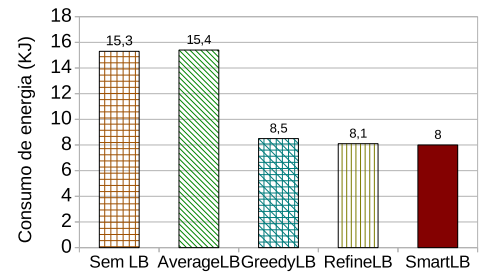

(a) $l b \_t e s t$

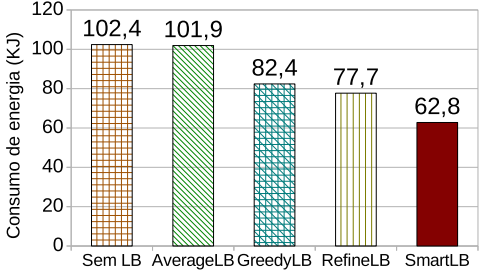

(b) kNeighbor

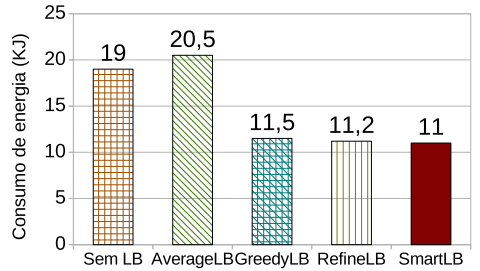

(c) ComprehensiveBench

Uma redução equivalente e significativa foi também observada no consumo de energia quando aplicado o SMARTLB com o benchmark kNeighbor. O consumo de energia foi reduzindo em 38,6\% em relação à execução sem balanceador, ou seja uma economia de $39,6 \mathrm{KJ}$ (de $102,4 \mathrm{KJ}$ para $62,8 \mathrm{KJ})$.

O tempo de execução e o consumo total de energia das execuções também foi reduzido quando o SMARTLB foi empregado na execução do benchmark ComprehensiveBench. O tempo total de execução apresentou uma redução de $41,8 \%$ em relação à execução sem balanceador. Para este benchmark, o uso da estratégia apresentou desempenho em média de $3,1 \%$ melhor que os alcançado pelos balanceador GREEDYLB e REFINELB. Ganhos equivalentes foram observados no consumo de energia quando aplicado a nossa proposta.

Na Figura 3 são apresentados os desbalanceamentos de carga mensurados a cada chamada do balanceador de carga proposto. Em todos os testes, o SMARTLB conseguiu concluir a execução dos benchmarks apresentando o menor de desbalanceamento. 
Figura 3. Desbalanceamento mensurado durante a execução dos benchmarks

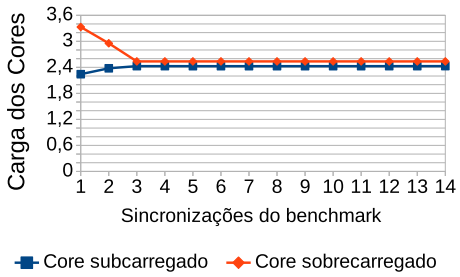

(a) $l b \_t e s t$

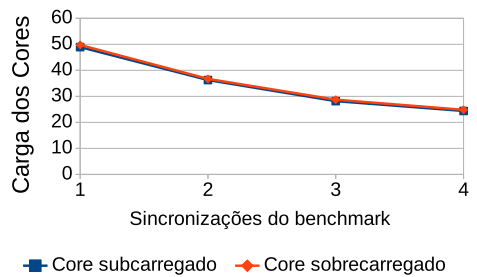

(b) kNeighbor

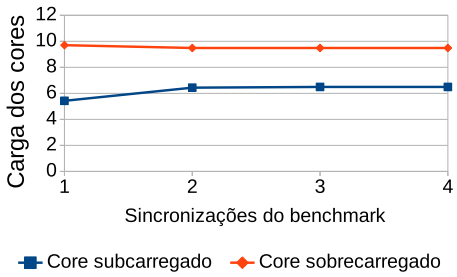

(c) ComprehensiveBench

Nos testes com o lb_test, na primeira chamada do balanceador a diferenças entre o core mais carregado e o core menos carregado era de 3,34 unidades. O SMARTLB conseguiu reduzir, até a última sincronização, essas diferenças para 0,12 . Nos testes realizados com os benchmarks kNeighbor e ComprehensiveBench, o desbalanceamentos iniciais eram menores, de 0,10 e 0, 28 unidades. Mesmo assim, com a utilização do SMARTLB, os desbalanceamentos foram reduzidos, finalizando a execução com 0,07 e 0,11 respectivamente.

\section{Conclusões e trabalhos futuros}

Este trabalho apresentou a proposta de um novo balanceador de carga denominado SMARTLB. Nossos resultados demonstram que o uso do balanceador de carga SMARTLB reduziu o tempo de execução e a quantidade de energia gasta quando aplicado em aplicações iterativas. O tempo total de execução e o consumo de energia foram reduzido em média $43,5 \%$ e $42,8 \%$ nos testes executados com três benchmarks. Os resultados alcançado pela nossa estratégia proposta foram melhores que os alcançados pelos balanceadores GREEDYLB e REFINELB

Como futuros trabalhos, pretende-se realizar melhorias no algoritmo de tomada de decisão do SMARTLB de modo a melhorar o controle de migrações de tarefas. Pretende-se também realizar testes em sistemas paralelos maiores utilizando problemas reais de computação científica bem como comparar com outros balanceadores de carga do estado da arte.

\section{Agradecimentos}

Trabalho apoiado por CNPq e CAPES com recursos do programa EU H2020 e do MCTI/RNPBrasil sob o projeto HPC4E de número 689772 e do edital de bolsa PIBIC da UNIJUI.

\section{Referências}

Cybenko, G. (1989). Dynamic load balancing for distributed memory multiprocessors. Journal of parallel and distributed computing, 7(2):279-301.

Padoin, E. L., Pilla, L. L., Castro, M., Boito, F. Z., Navaux, P. O. A., and Mehaut, J.-F. (2014). Performance/energy trade-off in scientific computing: The case of ARM big.LITTLE and Intel Sandy Bridge. IET Computers \& Digital Techniques, 2(3):1-14.

Pilla, L. L., Bozzetti, T. C., Castro, M., Navaux, P. O. A., and Méhaut, J.-F. (2015). Comprehensivebench: a benchmark for the extensive evaluation of global scheduling algorithms. Journal of Physics: Conference Series, 649(1):012007.

Zheng, G., Meneses, E., Bhatele, A., and Kale, L. V. (2010). Hierarchical load balancing for charm++ applications on large supercomputers. In Parallel Processing Workshops (ICPPW), 2010 39th International Conference on, pages 436-444. IEEE. 